T. Murai

Nagoya Math. J.

Vol. 122 (1991), 19-42

\title{
ANALYTIC CAPACITY FOR TWO SEGMENTS
}

\author{
TAKAFUMI MURAI
}

\section{$\S 1$. Introduction}

The analytic capacity $\gamma(E)$ of a compact set $E$ in the complex plane $\mathbf{C}$ is defined by $\gamma(E)=\sup \left|f^{\prime}(\infty)\right|$, where $-f^{\prime}(\infty)$ is the $1 / z$-coefficient of $f(\zeta)$ at infinity and the supremum is taken over all bounded analytic functions $f(\zeta)$ outside $E$ with supremum norm less than or equal to 1 . Analytic capacity $\gamma(\cdot)$ plays various important roles in the theory of bounded analytic functions.

It is known that $\gamma(E) \leq|E|$, where $|\cdot|$ is the (generalized) length (i.e., the 1-dimension Hausdorff measure [3, CHAP. III]) and that the inverse relation does not exist, in general. In fact, Vitushkin [14] constructs an example of a set with positive length but zero analytic capacity, and Garnett [3, p. 87] also points out that the planar Cantor set with ratio $1 / 4$

$$
E(1 / 4)=\bigcap_{n=0}^{\infty} E_{n}
$$

satisfies the same property. Here $E_{0}$ is the unit square $[0,1] \times[0,1]$ and $E_{n}$ is inductively defined from $E_{n-1}$ with each square $Q$ of $E_{n-1}$ replaced by four squares with sides $4^{-n}$ in the four corners of $Q$. The set $E_{n}$ is a union of $4^{n}$ squares with sides $4^{-n}$, and the projections of these $4^{n}$ squares to the line $\mathscr{L}: y=x / 2$ do not mutually overlap. Hence if we choose $\mathscr{L}$ as a new axis, then $E_{n}$ seems like a discontinuous graph. From this point of view, the author [8, CHAP. III] defined cranks and studied their analytic capacities: Cranks are nothing but deformations of sets of Vitushkin-Garnett type, however, these discontinuous graphs simplify the computation of analytic capacity and enable us to construct various examples [8, Theorem F], [9]. Hence clarifying the geometric meaning of cranks is important and would be applicable to study analytic capacities of general sets. (Cranks are closely related to fractals (Mandelbrot [6]).)

Received April 21, 1989. 
Here are simple cranks of degree 1:

$$
\Gamma(1+i y)=[-1 / 2,1 / 2] \cup(1+i y+[-1 / 2,1 / 2]) \quad(y>0) .
$$

This is a subclass of

$$
\Gamma(z)=[-1 / 2,1 / 2] \cup(z+[-1 / 2,1 / 2]) \quad(z \in \mathbf{C}),
$$

where, in general, $(z+w E)=\{z+w \zeta ; \zeta \in E\} \quad(z, w \in \mathbf{C} ; E \subset \mathbf{C})$. The purpose of this note is to study $\gamma(z)=\gamma(\Gamma(z))(z \in \mathbf{C})$ and show a role of cranks $\Gamma(1+i y)(y>0)$ in an extremum problem.

In fluid dynamics, $\Gamma(z)$ is a model of biplane wing sections, and the study of flows obstructed by $\Gamma(z)$ is classical (Ferrari [1], Garrick [3]). As is well known, there exists uniquely an analytic function $f_{z}(\zeta)$ outside $\Gamma(z)$ such that

(1) $f_{z}(\zeta)$ is integrable on $\partial \Gamma(z)^{\dagger)}$ (with respect to the length element $|d \zeta|), f_{z}(\zeta)$ is real-valued continuous on $\partial \Gamma(z)$ and $f_{z}(\infty)=-i$,

(2) $\left|f_{z}(p)\right|$ exists at the right endpoint $p$ of each component of $\Gamma(z)$ (Joukowksi's hypothesis).

Here $\partial \Gamma(z)$ is the subboundary of $\Gamma(z)^{c}$ which corresponds to $\Gamma(z)-$ \{endpoints of $\Gamma(z)$ \} topologically; $\partial \Gamma(z)$ has two sides. Condition (1) means that $f_{z}(\zeta)$ is a velocity field obstructed by $\Gamma(z)$ with velocity $i$ at infinity, and (2) means that vortexes at endpoints of $\Gamma(z)$ are negligible. We define the lift coefficient for $\Gamma(z)$ by

$$
\mathscr{L}(z)=\frac{1}{4}\left|\frac{1}{2 \pi} \int_{\partial \Gamma(z)} f_{z}(\zeta)^{2} d \zeta\right|\left(=\frac{1}{2}\left|f_{z}^{\prime}(\infty)\right|\right) .
$$

Using Blasius' theorem [7, p. 173], Kutta-Joukowski shows that $4 \pi \mathscr{L}(z) \sin \alpha$ gives the lift for $\Gamma(z)$ with respect to the velocity field with density 1 and velocity $e^{i \alpha}$ at infinity $(0 \leq \alpha \leq 2 \pi)$ (cf. [7, CHAP. VII], [3]). In the section 2 , we shall give a formula for $\gamma(z)$ in terms of $\mathscr{L}(z)$ and shall show that $\mathscr{L}(z) \leq \gamma(z)$ (Theorems 1 and 2). To compute $\gamma(z)$ practically, it is necessary to study the so-called modulus-invariant arcs. In the section 2 , we shall show two lemmas (with respect to modulus-invariant arcs) which will be used later. Using our formula along modulus-invariant arcs, we shall show, in the section 4 , that the behaviour of $\gamma(z)$ near 1 is critical (Theorem 8). In the section 5 , we shall show that

$$
\sigma_{0}=\min _{y \geq 0} \gamma(1+i y) / \gamma(1),
$$

where $\sigma_{0}$ is defined by the infimum of $\gamma(x+i y) / \gamma(x)$ over all real numbers

†) The condition " $\lim _{\varepsilon \downarrow 0} \int_{|\zeta-p|=\varepsilon}\left|f_{z}\right||d \zeta|=0(p= \pm 1 / 2, z \pm 1 / 2)$ " is required. 
$x$ and $y$ (Theorem 13). Since $\gamma(z)=1 / 2,2 \sigma_{0}$ equals the minimum of analytic capacities of cranks $\Gamma(1+i y)(y>0)$. This shows that the computation of $r(1+i y)(y>0)$ is essential in this extremum problem. We shall also show a practical method to estimate $\sigma_{0}$. Theorem 13 suggests that $E(1 / 4)$ is an extreme in a sense. Our method works for unions of two segments with different length, however, this is not applicable to unions of three segments.

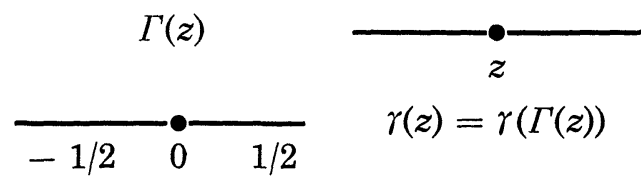

\section{§2. A formula for $\gamma(z)$}

In this section, we give a formula for $\gamma(z)(z \in \mathbf{C})$. Without loss of generality, we may assume that $z$ is contained in $P=\{\zeta \in \mathbf{C} ; \operatorname{Re} \zeta \geq 0$, $\operatorname{Im} \zeta \geq 0\}$, where $\operatorname{Re} \zeta$ and $\operatorname{Im} \zeta$ are the real part and the imaginary part of $\zeta$, respectively. A domain $\Gamma(z)^{c}$ is univalently mapped onto a ring $\left\{\zeta \in \mathbf{C} ; r<|\zeta|<r^{\prime}\right\}$. The modulus of $\Gamma(z)^{c}$ is defined by $\bmod \left(\Gamma(z)^{c}\right)=r^{\prime} \mid r$ [12, p. 199]. An arc $\lambda$ in $P$ is called modulus-invariant, if $\bmod \left(\Gamma(z)^{c}\right)$ is a constant on $\lambda$. For $z \in P, \operatorname{Im} z>0, \lambda(z)$ denotes the modulus-invariant arc in $P$ with endpoints $z$ and a real number; this real number is uniquely determined by $z$ and larger than 1 . In this section, we show the following two theorems.

Theorem 1. For $z \in P, \operatorname{Im} z>0$,

$$
\gamma(z)=\frac{1}{2}+\frac{\operatorname{Im} z}{2} \int_{\lambda(z)}\left\{\frac{\gamma(\zeta)}{\mathscr{L}(\zeta)}-1\right\} \frac{d(\operatorname{Im} \zeta)}{(\operatorname{Im} \zeta)^{2}}
$$

where $z$ is chosen as the initial point of this curvilinear integral.

TheOREM 2. $\mathscr{L}(z) \leq \gamma(z)(z \in P)$. Equality holds if and only if $z$ is real.

Since $z$ is the initial point of the integral in (3), Theorems 1 and 2 show that $\gamma(z)<1 / 2(z \in P, \operatorname{Im} z>0)$. Here are some lemmas necessary for the proof. The following lemma is a version of biplane theory to analytic capacity (Ferrari [1], Garrick [3], Sasaki [13, pp. 208-213]).

Lemma 3. For $0<k<1$ and $t \geq 0$, we define 
(4) $\xi_{k}(t)=\left[\frac{2 m_{k}^{2}+\left(1+k^{2}\right) t^{2}-\sqrt{\left\{2 m_{k}^{2}+\left(1+k^{2}\right) t^{2}\right\}^{2}-4\left(1+k^{2} t^{2}\right)\left(m_{k}^{4}+t^{2}\right)}}{2\left(1+k^{2} t^{2}\right)}\right]^{1 / 2}$,

$$
\begin{aligned}
& \eta_{k}(t)=\left[\frac{2 m_{k}^{2}+\left(1+k^{2}\right) t^{2}+\sqrt{\left\{2 m_{k}^{2}+\left(1+k^{2}\right) t^{2}\right\}^{2}-4\left(1+k^{2} t^{2}\right)\left(m_{k}^{4}+t^{2}\right)}}{2\left(1+k^{2} t^{2}\right)}\right]^{1 / 2}, \\
& l_{k}(t)=\tau_{k}+\int_{0}^{t}\left\{\eta_{k}(s)-\xi_{k}(s)\right\} d s,
\end{aligned}
$$

where

$$
\begin{gathered}
m_{k}=\frac{1}{k} \sqrt{\frac{E\left(k^{\prime}\right)}{K\left(k^{\prime}\right)}}, \quad \tau_{k}=2 \int_{1}^{m_{k}} \frac{m_{k}^{2}-s^{2}}{\sqrt{s^{2}-1} \sqrt{1-k^{2} s^{2}}} d s \\
E\left(k^{\prime}\right)=\int_{0}^{1} \sqrt{\frac{1-k^{\prime 2} s^{2}}{1-s^{2}}} d s, \quad K\left(k^{\prime}\right)=\int_{0}^{1} \frac{d s}{\sqrt{1-s^{2}} \sqrt{1-k^{\prime 2} s^{2}}}, \quad k^{\prime}=\sqrt{1-k^{2}} .
\end{gathered}
$$

Let

$$
\begin{aligned}
z_{k}(t) & =x_{k}(t)+i y_{k}(t) \\
& =1+\left\{-\tau_{k}+2 \int_{0}^{t} \xi_{k}(s) d s+\frac{i \pi}{k^{2} K\left(k^{\prime}\right)}\right\} / l_{k}(t) .
\end{aligned}
$$

Then

$$
\gamma\left(z_{k}(t)\right)=\left\{\frac{1-k}{2 k} \sqrt{t^{2}+k^{-2}}\right\} / l_{k}(t) .
$$

Proof. Since this lemma plays an important role in the proof of Theorems 1 and 2, we give the proof of this lemma, for the sake of completeness. For $0<k<1$ and $t \geq 0$, we write $\xi=\xi_{k}(t)$ and $\eta=\eta_{k}(t)$. Take a Schwarz-Christoffel transformation

$$
f(\zeta)=\int_{0}^{\zeta} \frac{s^{2}-m_{k}^{2}}{\sqrt{s-1} \sqrt{s+1} \sqrt{k s-1} \sqrt{k s+1}} d s-i t \zeta,
$$

where we choose a branch of the square root so that the upper half plane is mapped to the positive orthant. Since

$$
m_{k}^{2}=\int_{1}^{1 / k} \frac{s^{2} d s}{\sqrt{s^{2}-1} \sqrt{1-k^{2} s^{2}}} / \int_{1}^{1 / k} \frac{d s}{\sqrt{s^{2}-1} \sqrt{1-k^{2} s^{2}}} .
$$

$f(\zeta)$ univalently maps $\{[-1 / k,-1] \cup[1,1 / k]\}^{c}$ onto $\left\{\left(-a+i\left[\alpha_{-}, \beta_{-}\right]\right) \cup\right.$ $\left.\left(a+i\left[\alpha_{+}, \beta_{+}\right]\right)\right\}^{c}$ for some $a>0, \alpha_{ \pm}<\beta_{ \pm}$. (See [13, pp. 208-213].) Pommerenke [11] shows that $\gamma(E)=|E| / 4$ if $E$ is a compact set on the real line. Since 


$$
\lim _{\zeta \rightarrow \infty} f(\zeta) / \zeta=(1 / k)-\text { it , }
$$

the conformal invariance of $\gamma(\cdot)$ and Pommerenke's theorem show that

$$
\begin{aligned}
\gamma((- & \left.\left.a+i\left[\alpha_{-}, \beta_{-}\right]\right) \cup\left(a+i\left[\alpha_{+}, \beta_{+}\right]\right)\right) \\
= & \left|\frac{1}{k}-i t\right| \gamma([-1 / k,-1] \cup[1,1 / k])=\frac{1-k}{2 k} \sqrt{t^{2}+k^{-2}} .
\end{aligned}
$$

Legendre's formula

$$
E(k) K\left(k^{\prime}\right)+E\left(k^{\prime}\right) K(k)-K(k) K\left(k^{\prime}\right)=\pi / 2 \quad[4, \text { p. 291] }
$$

shows that

$$
2 a=2 \operatorname{Re} f(1)=2 \int_{0}^{1} \frac{m_{k}^{2}-s^{2}}{\sqrt{1-s^{2}} \sqrt{1-k^{2} s^{2}}} d s=\frac{\pi}{k^{2} K\left(k^{\prime}\right)} .
$$

Let

$$
\psi_{k}(x)=\int_{1}^{x} \frac{m_{k}^{2}-s^{2}}{\sqrt{s^{2}-1} \sqrt{1-k^{2} s^{2}}} d s \quad(1 \leq x \leq 1 / k) .
$$

Then (4) and (5) show that

$$
1<\xi<m_{k}, \quad \psi_{k}^{\prime}(\xi)=t ; \quad m_{k}<\eta<1 / k, \quad \psi_{k}^{\prime}(\eta)=-t .
$$

These inequalities yield that

$$
\beta_{+}=\psi_{k}(\xi)-t \xi, \quad \alpha_{+}=-\psi_{k}(\eta)-t \eta, \alpha_{-}=-\beta_{+},
$$

and hence

$$
\begin{aligned}
& \beta_{+}-\alpha_{+}=\psi_{k}(\eta)+\psi_{k}(\xi)+t(\eta-\xi), \\
& \alpha_{-}-\beta_{+}=2 t \xi-2 \psi_{k}(\xi) .
\end{aligned}
$$

Rotating, translating and normalizing $\left(-a+i\left[\alpha_{-}, \beta_{-}\right]\right) \cup\left(a+i\left[\alpha_{+}, \beta_{+}\right]\right)$, we obtain

$$
\begin{aligned}
\gamma\left(z_{k}^{*}(t)\right) & =\frac{1-k}{2 k} \sqrt{t^{2}+k^{-2}} \frac{1}{\psi_{k}(\eta)+\psi_{k}(\xi)+t(\eta-\xi)}, \\
z_{k}^{*}(t) & =1+\frac{2 t \xi-2 \psi_{k}(\xi)+i \pi /\left\{k^{2} K\left(k^{\prime}\right)\right\}}{\psi_{k}(\eta)+\psi_{k}(\xi)+t(\eta-\xi)} .
\end{aligned}
$$

Since

$$
\frac{d}{d t}\left\{\psi_{k}\left(\xi_{k}(t)\right)-t \xi_{k}(t)\right\}=-\xi_{k}(t), \quad \psi_{k}\left(\xi_{k}(0)\right)=\tau_{k} / 2
$$


we have

$$
\psi_{k}\left(\xi_{k}(t)\right)-t \xi_{k}(t)=\frac{\tau_{k}}{2}-\int_{0}^{t} \xi_{k}(s) d s
$$

In the same manner,

$$
\psi_{k}\left(\eta_{k}(t)\right)+\operatorname{t\eta }_{k}(t)=\frac{\tau_{k}}{2}+\int_{0}^{t} \eta_{k}(s) d s
$$

Thus

$$
\psi_{k}\left(\eta_{k}(t)\right)+\psi_{k}\left(\xi_{k}(t)\right)+t\left\{\eta_{k}(t)-\xi_{k}(t)\right\}=l_{k}(t), \quad z_{k}^{*}(t)=z_{k}(t),
$$

which yields (6).

Lemma 4 (the lift formula). The function $\mathscr{L}(z)$ is continuous on $P$ and

$$
\mathscr{L}\left(z_{k}(t)\right)=\left\{k t+\frac{1}{k t}\right\} \frac{\eta_{k}(t)-\xi_{k}(t)}{2 k l_{k}(t)} \quad(0<k<1, t>0) .
$$

This lemma is known in fluid dynamics ([1], [3], [13, p. 213]). The outline of the proof is as follows. For $0<k<1$ and $t>0$, let $f(\zeta)$ be the Schwarz-Christoffel transformation used in the proof of Lemma 3. Then if $(\zeta)$ univalently maps $\{[-1 / k,-1] \cup[1,1 / k]\}^{c}$ onto a domain similar to $\Gamma\left(z_{k}(t)\right)^{c}$, say $R$. For real numbers $U, V, \rho, n$, we take

$$
\Omega(\zeta)=U \zeta-i V \int_{0}^{\zeta} \frac{s^{2}-m_{k}^{2}}{\sqrt{s^{2}-1} \sqrt{k^{2} s^{2}-1}} d s-i \rho \int_{0}^{\zeta} \frac{s-n}{\sqrt{s^{2}-1} \sqrt{k^{2} s^{2}-1}} d s .
$$

Then $\frac{d}{d w} \Omega(h(w))$ is an analytic function in $R$, where $h(w)$ is the inverse function of if( $\zeta)$. Using Joukowski's hypothesis and (the argument of $\left.\frac{d}{d w} \Omega(h(\infty))\right)=-\pi / 2$, we determine $U, V, \rho, n$. Translating and normalizing $R$, we obtain $f_{z_{k}(t)}(\zeta)$. Computing $f_{z_{k}(t)}^{\prime}(\infty)$, we obtain (10).

Lemma 5. $\frac{\tau_{k}}{2}=\int_{0}^{\infty}\left\{\frac{1}{k}-\eta_{k}(s)\right\} d s=\int_{0}^{\infty}\left\{\xi_{k}(s)-1\right\} d s \quad(0<k<1)$.

Proof. Since

$$
\frac{1}{k}-\eta_{k}(t)=O\left(t^{-2}\right), \quad \xi_{k}(s)-1=O\left(t^{-2}\right) \quad(t \longrightarrow \infty),
$$

two integrals in the required equalities converge. Equality (8) shows that 


$$
\int_{0}^{t}\left\{\frac{1}{k}-\eta_{k}(s)\right\} d s=\frac{\tau_{k}}{2}-\psi_{k}\left(\eta_{k}(t)\right)+t\left\{\frac{1}{k}-\eta_{k}(t)\right\}
$$

Letting $t$ tend to infinity, we obtain

$$
\int_{0}^{\infty}\left\{\frac{1}{k}-\eta_{k}(s)\right\} d s=\frac{\tau_{k}}{2}-\psi_{k}(1 / k)=\frac{\tau_{k}}{2} .
$$

Thus the first equality holds. Analogously, (7) yields the second equality.

In order to prove Theorems 1 and 2 , it is necessary to use the following property:

(11) To $z \in P, \operatorname{Im} z>0$, there corresponds uniquely a pair $(k, t)$ so that $z_{k}(t)=z$ and $\lambda(z)=\left\{z_{k}(s) ; s \geq t\right\} \cup\{(1+k) /(1-k)\}$.

This property will be shown in the next section. Here we give the proof of Theorems 1 and 2, assuming (11). First we give the proof of Theorem 1. For $z \in P, \operatorname{Im} z>0$, let $(k, t)$ be the pair in (11). Equality (10) shows that

$$
\begin{aligned}
y_{k}^{\prime}(s) & =-\frac{\pi}{k^{2} K\left(k^{\prime}\right)} \frac{l_{k}^{\prime}(s)}{l_{k}(s)^{2}}=-\frac{\eta_{k}(s)-\xi_{k}(s)}{l_{k}(s)} y_{k}(s) \\
& =\frac{2 k^{2} s}{1+k^{2} s^{2}} \mathscr{L}\left(z_{k}(s)\right) y_{k}(s) \quad(s>0) .
\end{aligned}
$$

Thus we have, by Lemmas 4, 5, (6) and (10),

$$
\begin{aligned}
& \frac{\gamma(z)-1 / 2}{\operatorname{Im} z}=\frac{\gamma\left(z_{k}(t)\right)-1 / 2}{y_{k}(t)}=\frac{k^{2} K\left(k^{\prime}\right) l_{k}(t)}{2 \pi}\left\{2 \gamma\left(z_{k}(t)\right)-1\right\} \\
& =\frac{k^{2} K\left(k^{\prime}\right)}{2 \pi}\left\{\frac{1-k}{k} \sqrt{t^{2}+k^{-2}}-\tau_{k}-\int_{0}^{t}\left(\eta_{k}(s)-\xi_{k}(s)\right) d s\right\} \\
& =\frac{k^{2} K\left(k^{\prime}\right)}{2 \pi}\left[\frac{1-k}{k}\left\{\sqrt{t^{2}+k^{-2}}-t\right\}-\tau_{k}+\int_{0}^{t}\left\{\frac{1}{k}-1-\eta_{k}(s)+\xi_{k}(s)\right\} d s\right] \\
& =\frac{k^{2} K\left(k^{\prime}\right)}{2 \pi}\left[\int_{t}^{\infty}\left\{\frac{1}{k}-1-\frac{(1-k) s}{\sqrt{1+k^{2} s^{2}}}\right\} d s-\int_{t}^{\infty}\left\{\frac{1}{k}-1-\eta_{k}(s)+\xi_{k}(s)\right\} d s\right] \\
& =-\frac{k^{2} K\left(k^{\prime}\right)}{2 \pi} \int_{t}^{\infty} \frac{2 k^{2} s}{1+k^{2} s^{2}}\left\{\frac{1-k}{2 k} \sqrt{s^{2}+k^{-2}}-\frac{1+k^{2} s^{2}}{2 k^{2} s}\left(\eta_{k}(s)-\xi_{k}(s)\right)\right\} d s \\
& =-\frac{1}{2} \int_{t}^{\infty} \frac{2 k^{2} s}{1+k^{2} s^{2}}\left\{\gamma\left(z_{k}(s)\right)-\mathscr{L}\left(z_{k}(s)\right)\right\} \frac{1}{y_{k}(s)} d s \\
& =\frac{1}{2} \int_{t}^{\infty} \frac{\gamma\left(z_{k}(s)\right)-\mathscr{L}\left(z_{k}(s)\right)}{\mathscr{L}\left(z_{k}(s)\right)} \frac{y_{k}^{\prime}(s)}{y_{k}(s)^{2}} d s=\frac{1}{2} \int_{\lambda(z)}\left\{\frac{\gamma(\zeta)}{\mathscr{L}(\zeta)}-1\right\} \frac{d(\operatorname{Im} \zeta)}{(\operatorname{Im} \zeta)^{2}} .
\end{aligned}
$$


This completes the proof of Theorem 1. Next we give the proof of Theorem 2. For $z \in P, \operatorname{Re} z>0, \operatorname{Im} z>0$, let $(k, t)$ be the pair in (11). We write $\xi=\xi_{k}(t)$ and $\eta=\eta_{k}(t)$. Equalities (4) and (5) show that

$$
\begin{aligned}
(\eta-\xi)^{2} & =\eta^{2}+\xi^{2}-2 \eta \xi \\
& =\frac{1}{1+k^{2} t^{2}}\left\{2 m_{k}^{2}+\left(1+k^{2}\right) t^{2}-2 \sqrt{\left(1+k^{2} t^{2}\right)\left(m_{k}^{4}+t^{2}\right)}\right\} .
\end{aligned}
$$

Thus we have, by Lemmas 3 and 4,

$$
\begin{aligned}
\gamma(z)-\mathscr{L}(z)=\frac{\gamma(z)^{2}-\mathscr{L}(z)^{2}}{\gamma(z)+\mathscr{L}(z)} \\
=\frac{1}{\{\gamma(z)+\mathscr{L}(z)\} l_{k}(t)^{2}}\left\{\frac{(1-k)^{2}}{4 k^{4}}\left(1+k^{2} t^{2}\right)-\frac{\left(1+k^{2} t^{2}\right)^{2}}{4 k^{4} t^{2}}(\eta-\xi)^{2}\right\} \\
=\frac{1+k^{2} t^{2}}{4\{\gamma(z)+\mathscr{L}(z)\} l_{k}(t)^{2} k^{4} t^{2}}\left\{(1-k)^{2} t^{2}-\left(1+k^{2} t^{2}\right)(\eta-\xi)^{2}\right\} \\
=\frac{\gamma(z)^{2}}{\{\gamma(z)+\mathscr{L}(z)\}(1-k)^{2} t^{2}} \\
\\
\quad \times\left[(1-k)^{2} t^{2}-\left\{2 m_{k}^{2}+\left(1+k^{2}\right) t^{2}-2 \sqrt{\left(1+k^{2} t^{2}\right)\left(m_{k}^{4}+t^{2}\right)}\right\}\right] \\
=\frac{2 \gamma(z)^{2}}{\{\gamma(z)+\mathscr{L}(z)\}(1-k)^{2} t^{2}}\left\{\sqrt{\left(k t^{2}+m_{k}^{2}\right)^{2}+\left(k m_{k}^{2}-1\right)^{2} t^{2}}-\left(k t^{2}+m_{k}^{2}\right)\right\} .
\end{aligned}
$$

A simple calculation shows that $k m_{k}^{2}>1$. Thus $\mathscr{L}(z)<\gamma(z) \quad(z \in P$, $\operatorname{Re} z>0, \operatorname{Im} z>0$ ). If $\operatorname{Re} z=0$ and $\operatorname{Im} z>0$, then we have

$$
\gamma(z)-\mathscr{L}(z)=\frac{\gamma(z)^{2}\left(k m_{k}^{2}-1\right)^{2}}{\{\gamma(z)+\mathscr{L}(z)\}(1-k)^{2} m_{k}^{2}}>0,
$$

by (12) and the continuity of $\gamma(z)$ and $\mathscr{L}(z)$. We now show that

$$
\left.\gamma(z) \leq \mathscr{L}(z)+\frac{C}{\log (1 / \operatorname{Im}} z\right) \quad(z \in P, 0<\operatorname{Im} z<1 / 2)
$$

for some absolute constant $C$. By (12), we have, with two absolute constants $C_{1}$ and $C_{2}$,

$$
\begin{aligned}
\gamma(z)-\mathscr{L}(z) & \leq \frac{\gamma(z)^{2}\left(k m_{k}^{2}-1\right)^{2}}{\{\gamma(z)+\mathscr{L}(z)\}(1-k)^{2}\left(k t^{2}+m_{k}^{2}\right)} \leq \frac{\left(k m_{k}^{2}-1\right)^{2}}{(1-k)^{2}\left(k t^{2}+m_{k}^{2}\right)} \\
& \leq \frac{k^{2} m_{k}^{4}}{(1-k)^{2} m_{k}^{2}}=\frac{E\left(k^{\prime}\right)}{(1-k)^{2} K\left(k^{\prime}\right)} \leq \frac{C_{1}}{(1-k)^{2} \log (1+(1 / k))}
\end{aligned}
$$

and 


$$
\begin{aligned}
\gamma(z)-\mathscr{L}(z) & \leq \frac{\left(k m_{k}^{2}-1\right)^{2}}{(1-k)^{2}\left(k t^{2}+m_{k}^{2}\right)}=\frac{k\left(k m_{k}^{2}-1\right)^{2}}{(1-k)^{2}\left(k^{2} t^{2}+k m_{k}^{2}\right)} \\
& \leq \frac{k^{3} m_{k}^{4}}{(1-k)^{2}\left(1+k^{2} t^{2}\right)}=\frac{m_{k}^{4}}{4 \gamma(z)^{2} k l_{k}(t)^{2}}=\frac{k^{3} m_{k}^{4} K\left(k^{\prime}\right)^{2} y_{k}(t)^{2}}{4 \pi^{2} \gamma(z)^{2}} \\
& =\frac{E\left(k^{\prime}\right)^{2}(\operatorname{Im} z)^{2}}{4 \pi^{2} \gamma(z)^{2} k} \leq C_{2}(\operatorname{Im} z)^{2} / k
\end{aligned}
$$

where $(k, t)$ is the pair associated with $z$. Thus

$$
\gamma(z)-\mathscr{L}(z) \leq \min \left\{\frac{C_{1}}{(1-k)^{2} \log (1+(1 / k))}, C_{2}(\operatorname{Im} z)^{2} / k\right\} .
$$

If $\operatorname{Im} z \leq k$, then $\gamma(z)-\mathscr{L}(z) \leq C_{2} \operatorname{Im} z$. If $\operatorname{Im} z>k$, then

$$
\gamma(z)-\mathscr{L}(z) \leq \frac{C_{1}}{(1-k)^{2} \log (1+(1 / k))} \leq \frac{C_{3}}{\log (1 / \operatorname{Im} z)}
$$

for some absolute constant $C_{3}$, because of $0<\operatorname{Im} z<1 / 2$. Thus

$$
\gamma(z)-\mathscr{L}(z) \leq \max \left\{\frac{C_{3}}{\log (1 / \operatorname{Im} z)}, C_{2} \operatorname{Im} z\right\},
$$

which gives (14). Since $\gamma(z)$ and $\mathscr{L}(z)$ are continuous on $P$, (14) shows that the equality holds for real numbers $z$. This completes the proof of Theorem 2.

Inequality (13) yields that

$$
\gamma(i y)-\mathscr{L}(i y) \geq C_{4} y \quad(0<y<1 / 2)
$$

for some absolute constant $C_{4}$. We do not know whether the order $\frac{1}{\log (1 / \operatorname{Im} z)}$ in (14) is best possible or not.

\section{§ 3. Modulus-invariant arcs}

To compute $\gamma(z)$ practically, it is necessary to study modulus-invariant arcs. To use later, we prepare, in this section, the following two lemmas; (15) and (16) in Lemma 6 give (11) which was used in the proof of Theorems 1 and 2 .

\section{Lemma 6.}

(15) $z_{k}(t)$ is a continuous homeomorphism from $Q=\{(k, t) ; 0<k<1$, $t \geq 0\}$ to $P-[0, \infty)$. 
(16) For $(k, t) \in Q, \lambda\left(z_{k}(t)\right)=\left\{z_{k}(s) ; s \geq t\right\} \cup\{(1+k) /(1-k)\}$.

(17) For $0<k<1, x_{k}(t)$ is strictly increasing, and $y_{k}(t)$ is strictly decreasing with respect to $t$.

Lemma 7. Let $a \geq 0$. Then, for any $k$ satisfying $k_{a}<k<1 \quad\left(k_{a}=\right.$ $\max \{(a-1) /(a+1), 0\})$, there exists uniquely $t_{a, k}>0$ such that $x_{k}\left(t_{a, k}\right)=a$. We have

(18) $y_{k}\left(t_{a, k}\right)$ is continuous and strictly increasing with respect to $k$.

(19) $\lim _{k \rightarrow k_{a}} y_{k}\left(t_{a, k}\right)=0$.

(20) $\quad a \tau_{k}=\int_{0}^{t_{a, k}}\left\{(1-a) \eta_{k}(s)+(1+a) \xi_{k}(s)\right\} d s$.

Proof of Lemma 6. For $0<k<1$, we have

$$
\left\{\begin{array}{l}
x_{k}(0)=0, \quad \lim _{t \rightarrow \infty} x_{k}(t)=\frac{1+k}{1-k}, \\
y_{k}(0)=\frac{\pi}{k^{2} K\left(k^{\prime}\right) \tau_{k}}, \quad \lim _{t \rightarrow \infty} y_{k}(t)=0 .
\end{array}\right.
$$

In fact, (4) and (5) show that

$$
\lim _{t \rightarrow \infty} \eta_{k}(t)=1 / k, \quad \lim _{t \rightarrow \infty} \xi_{k}(t)=1,
$$

and hence

$$
\begin{aligned}
\lim _{t \rightarrow \infty} x_{k}(t) & =1+2 \lim _{t \rightarrow \infty} \int_{0}^{t} \xi_{k}(s) d s / \int_{0}^{t}\left\{\eta_{k}(s)-\xi_{k}(s)\right\} d s \\
& =1+\frac{2}{(1 / k)-1}=\frac{1+k}{1-k} .
\end{aligned}
$$

The other three equalities in (21) are easily seen. We have

$$
\lim _{k \rightarrow 0} y_{k}(0)=0, \quad \lim _{k \rightarrow 1} x_{k}\left(1 / k^{\prime}\right)=\lim _{k \rightarrow 1} y_{k}\left(1 / k^{\prime}\right)=\infty .
$$

In fact, we have

$$
\begin{aligned}
\lim _{k \rightarrow 0} k^{2} \tau_{k} & =2 \lim _{k \rightarrow 0} k^{2} \int_{1}^{m_{k}} \frac{m_{k}^{2}-s^{2}}{\sqrt{s^{2}-1} \sqrt{1-k^{2} s^{2}}} d s \\
& =2 \lim _{k \rightarrow 0} k^{2} m_{k}^{2} \log m_{k}=\lim _{k \rightarrow 0} \frac{E\left(k^{\prime}\right)}{K\left(k^{\prime}\right)} \log \left\{\frac{E\left(k^{\prime}\right)}{k^{2} K\left(k^{\prime}\right)}\right\}=2,
\end{aligned}
$$

which gives 


$$
\lim _{k \rightarrow 0} y_{k}(0)=\lim _{k \rightarrow 0} \frac{\pi}{k^{2} K\left(k^{\prime}\right) \tau_{k}}=\frac{\pi}{2} \lim _{k \rightarrow 0} \frac{1}{K\left(k^{\prime}\right)}=0
$$

Since $\lim _{k \rightarrow 1} m_{k}=1$, we have, with $n_{k}=\sqrt{1-k^{2} m_{k}^{2}} / k^{\prime}$,

$$
\begin{aligned}
\lim _{k \rightarrow 1} \tau_{k} & =2 \lim _{k \rightarrow 1}\left\{m_{k}^{2} \int_{n_{k}}^{1} \frac{d s}{\sqrt{1-s^{2}} \sqrt{1-k^{\prime 2} s^{2}}}-k^{-2} \int_{n_{k}}^{1} \sqrt{\frac{1-k^{\prime 2} s^{2}}{1-s^{2}}} d s\right\} \\
& =2 \lim _{k \rightarrow 1}\left(m_{k}^{2}-k^{-2}\right) \int_{n_{k}}^{1} \frac{d s}{\sqrt{1-s^{2}}}=0 .
\end{aligned}
$$

Recall that $\xi_{k}(s)>1,0<\eta_{k}(s)-\xi_{k}(s)<(1 / k)-1$. We have

$$
\begin{aligned}
\liminf _{k \rightarrow 1} x_{k}\left(1 / k^{\prime}\right) & =1+\liminf _{k \rightarrow 1} 2 \int_{0}^{1 / k^{\prime}} \xi_{k}(s) d s / \int_{0}^{1 / k^{\prime}}\left\{\eta_{k}(s)-\xi_{k}(s)\right\} d s \\
& \geq 1+\liminf _{k \rightarrow 1} \frac{2}{(1 / k)-1}=\infty
\end{aligned}
$$

and

$$
\begin{aligned}
\liminf _{k \rightarrow 1} y_{k}\left(1 / k^{\prime}\right) & =\liminf _{k \rightarrow 1} \pi /\left\{k^{2} K\left(k^{\prime}\right) \int_{0}^{1 / k^{\prime}}\left(\eta_{k}(s)-\xi_{k}(s)\right) d s\right\} \\
& =\liminf _{k \rightarrow 1} \frac{2 k^{\prime}}{(1 / k)-1}=\infty .
\end{aligned}
$$

Thus (22) holds.

Since

$$
l_{k}^{\prime}(t)=\eta_{k}(t)-\xi_{k}(t)>0,
$$

$l_{k}(t)$ is strictly increasing, and hence $y_{k}(t)$ is strictly decreasing. Recall (7) and (9). Since

$$
x_{k}(t)=1+\frac{2}{l_{k}(t)}\left\{-\psi_{k}\left(\xi_{k}(t)\right)+t \xi_{k}(t)\right\},
$$

we have, with $\xi=\xi_{k}(t)$ and $\eta=\eta_{k}(t)$,

$$
\begin{aligned}
x_{k}^{\prime}(t) & =\frac{2}{l_{k}(t)^{2}}\left\{\xi l_{k}(t)-\left(-\psi_{k}(\xi)+t \xi\right)(\eta-\xi)\right\} \\
& =\frac{2}{l_{k}(t)^{2}}\left\{\xi \psi_{k}(\eta)+\eta \psi_{k}(\xi)\right\} .
\end{aligned}
$$

Since $\psi_{k}^{\prime}(t)>0\left(1<t<m_{k}\right)$, we have $\psi_{k}(\xi)>0$. Since $\psi_{k}^{\prime}(t)<0\left(m_{k}<t\right.$ $<1 / k)$, we have $\psi_{k}(\eta)>\psi_{k}(1 / k)=0$. Consequently, $x_{k}^{\prime}(t)>0$. Thus (17) holds. Inequalities (21) show that $\lim _{t \rightarrow \infty} z_{k}(t)=(1+k) /(1-k)$. Thus (17) 
yields (16). Let $W_{k}$ be the compact set bounded by the $x, y$ axes and $\lambda\left(i y_{k}(0)\right)$. Then (16) and (17) show that

$$
\begin{aligned}
& W_{k} \subset\left\{x+i y ; 0 \leq x \leq \frac{1+k}{1-k}, 0 \leq y \leq y_{k}(0)\right\}, \\
& W_{k} \supset\left\{x+i y ; 0 \leq x \leq x_{k}\left(1 / k^{\prime}\right), 0 \leq y \leq y_{k}\left(1 / k^{\prime}\right)\right\},
\end{aligned}
$$

and hence, by (22),

$$
\bigcap_{0<k<1} W_{k}=[0,1], \quad \bigcup_{0<k<1} W_{k}=P .
$$

This shows that $z_{k}(t)$ is an onto mapping from $Q$ to $P-[0, \infty)$. Recall that $\lambda\left(i y_{k}(0)\right)$ is a modulus-invariant arc with modulus $\bmod (\{[-1 / k,-1]$ $\left.\cup[1,1 / k]\}^{c}\right)$. The domain $\{[-1 / k,-1] \cup[1,1 / k]\}^{c}$ is univalently mapped onto a Grötzsch's domain $G_{p_{k}}=\{z \in \mathbf{C} ;|z|>1\}-\left[p_{k}, \infty\right)$ with

$$
p_{k}=1+\frac{8 k}{(1-k)^{2}}\left\{1+\frac{1+k}{2 \sqrt{k}}\right\} \text {. }
$$

Since $\bmod \left(G_{p}\right)$ is strictly increasing with respect to $p[5, \mathrm{p} .72]$ and $p_{k}$, $(1+k) /(1-k)\left(=\lim _{t \rightarrow \infty} z_{k}(t)\right)$ are strictly increasing with respect to $k$, we have

$$
W_{k} \subset W_{k^{\prime}}, \quad W_{k} \cap \lambda\left(i y_{k^{\prime}}(0)\right)=\varnothing \quad\left(k<k^{\prime}\right) .
$$

Notice that $z_{k}(t)$ is continuous on $Q$ (with respect to $(k, t)$ ). Since $(1+k) /(1-k)\left(=\lim _{t \rightarrow \infty} z_{k}(t)\right)$ is continuous with respect to $k$, we have $\bigcap_{k<\mu<1} W_{\mu}=W_{k}$. Thus (15) holds. This completes the proof of Lemma 6 .

Proof of Lemma 7. Let $\mu(a)=\{\zeta \in \mathbf{C} ; \operatorname{Re} \zeta=a\}(a \geq 0)$. Then Lemma 6 shows that

$$
\begin{array}{ll}
\mu(a) \cap \lambda\left(i y_{k}(0)\right)=\varnothing & \left(0<k<k_{a}\right), \\
\mu(a) \cap \lambda\left(i y_{k}(0)\right) \text { is a singleton } & \left(k_{a}<k<1\right) .
\end{array}
$$

Hence, if $k>k_{a}$, then, by (17), there exists uniquely $t_{a, k} \geq 0$ such that $z_{k}\left(t_{a, k}\right)$ is the unique element of $\mu(a) \cap \lambda\left(i y_{k}(0)\right)$. Evidently, $x_{k}\left(t_{a, k}\right)=a$. By (15) and (23), $y_{k}\left(t_{a, k}\right)$ is continuous and strictly increasing with respect to $k$. If $a>1$, then $k_{a}=(a-1) /(a+1)$, and hence (16) gives (19). If $0 \leq a \leq 1$, then $k_{a}=0$, and hence

$$
\limsup _{k \rightarrow k_{a}} y_{k}\left(t_{a, k}\right) \leq \lim _{k \rightarrow 0} y_{k}(0)=0 \text {. }
$$

Since 


$$
a=x_{k}\left(t_{a, k}\right)=1+\left\{-\tau_{k}+2 \int_{0}^{t_{a, k}} \xi_{k}(s) d s\right\} / l_{k}(t),
$$

we have (20). This completes the proof of Lemma 7.

\section{§4. Asymptotic behaviour of $\gamma(z)$}

In this section, we show

\section{TheOREM 8.}

$$
\begin{aligned}
\text { (24) } \quad r_{y}^{+}(0) & =+\infty, \\
\text { (25) } \quad r_{y}^{+}(a) & =\frac{1}{4 \pi} \log \frac{1}{a}(>0) \quad(0<a<1), \\
\text { (26) } \quad r_{y}^{+}(1) & <0, \\
\text { (27) } \quad r_{y}(a) & =0, \\
r_{y y}(a) & =-\frac{1}{8 \pi^{2}} \frac{a+1}{a-1}\left\{E\left(\frac{2 \sqrt{a}}{a+1}\right)-\frac{a-1}{a+1} K\left(\frac{2 \sqrt{a}}{a+1}\right)\right\}^{2} \\
& \quad(<0) \quad(a>1),
\end{aligned}
$$$$
\text { (27) } r_{y}(a)=0 \text {, }
$$

where $\gamma_{y}^{+}(a)=\lim _{y \downarrow 0}\{\gamma(a+i y)-\gamma(a)\} / y, \gamma_{y}=\partial \gamma / \partial y$ and $\gamma_{y y}=\partial^{2} \gamma / \partial y^{2}$.

Equalities (25)-(27) show that $\gamma_{y}^{+}(a)$ is discontinuous at $a=1$. We see that $\gamma_{y}(1)=1 /\left\{2 \pi \sqrt{c^{2}-1}\right\}=0.662 \cdots / 2 \pi$, where $c$ is the number satisfying $c / \sqrt{c^{2}-1}=\log \left(c+\sqrt{c^{2}-1}\right)$ (cf. Lemma 10). Since

$$
\gamma(1)=1 / 2, \quad \lim _{y \rightarrow \infty} \gamma(1+i y)=1 / 2,
$$

(26) shows that $\gamma(1+i y)$ has the minimum in $(0, \infty)$. If $0<a_{0}<1$ is sufficiently near to 1 , the behaviour of $\gamma\left(a_{0}+i y\right)(y>0)$ is more complicated. Let $y_{0}>0$ be a point such that $\gamma\left(1+i y_{0}\right)=\min _{y \geq 0} \gamma(1+i y)$. Since $\gamma\left(1+i y_{0}\right)<1 / 2$, we can choose $0<a_{1}<1$ so that $\max _{a_{1 \leq a \leq 1}} \gamma\left(a+i y_{0}\right)$ $\left(=\gamma_{0}\right.$, say) is less than $1 / 2$. If we choose $a_{0}$ so that $\max \left\{a_{1}, 1-2\left(1-2 \gamma_{0}\right)\right\}$ $<a_{0}<1$, then $\gamma\left(a_{0}+i y_{0}\right)<\gamma\left(a_{0}\right)$, and hence (25) shows that $\gamma\left(a_{0}+i y\right)$ has a local maximum in $\left(0, y_{0}\right)$. Since $\gamma\left(a_{0}+i y_{0}\right)<\gamma\left(a_{0}\right)$ and $\lim _{y \rightarrow \infty} \gamma\left(a_{0}+i y\right)$ $=1 / 2, \gamma\left(a_{0}+i y\right)$ has the minimum in $(0, \infty)$. Thus $\gamma\left(a_{0}+i y\right)$ has at least two extrema. A calculation shows that $\lim _{a \downarrow 1} \gamma_{y y}(a)=-\infty$ and

$$
\gamma_{y y}^{+}(1)=2 \lim _{y \downarrow 0}\left\{\gamma(1+i y)-\gamma(1)-y \gamma_{y}^{+}(y)\right\} / y^{2}=+\infty .
$$

Thus $\gamma_{y y}^{+}(a)(a \geq 1)$ is also discontinuous at $a=1$.

Here are some lemmas necessary for the proof. 
LEMMA 9. $\lim _{k \rightarrow 0} k t_{a, k}=\frac{2 \sqrt{a}}{1-a} \quad(0<a<1)$.

Proof. Equalities (4) and (5) show that, with $\xi_{a, k}=\xi_{k}\left(t_{a, k}\right)$ and $\eta_{a, k}=$ $\eta_{k}\left(t_{a, k}\right)$

$$
\begin{aligned}
& \frac{1-\xi_{a, k}^{2} m_{k}^{-2}}{\sqrt{\xi_{a, k}^{2}-1} \sqrt{1-k^{2} \xi_{a, k}^{2}}}=t_{a, k} m_{k}^{-2}, \\
& \frac{1-m_{k}^{2} \eta_{a, k}^{-2}}{\sqrt{1-\eta_{a, k}^{-2}} \sqrt{1-k^{2} \eta_{a, k}^{2}}}=t_{a, k} \eta_{a, k}^{-1} .
\end{aligned}
$$

Equality (20) shows that

$$
\begin{aligned}
0 & =-a \tau_{k}+\int_{0}^{t_{a, k}}\left\{(1-a) \eta_{k}(s)+(1+a) \xi_{k}(s)\right\} d s \\
& =(1-a)\left\{\frac{\tau_{k}}{2}+\int_{0}^{t_{a, k}} \eta_{k}(s) d s\right\}+(1+a)\left\{-\frac{\tau_{k}}{2}+\int_{0}^{t_{a, k}} \xi_{k}(s) d s\right\} \\
& =(1-a)\left\{\psi_{k}\left(\eta_{a, k}\right)+t_{a, k} \eta_{a, k}\right\}+(1+a)\left\{-\psi_{k}\left(\xi_{a, k}\right)+t_{a, k} \xi_{a, k}\right\}
\end{aligned}
$$

and hence

$$
\begin{aligned}
\eta_{a, k}^{-2}\left\{(1+a) \int_{1}^{\xi_{a, k}} \frac{m_{k}^{2}-s^{2}}{\sqrt{s^{2}-1} \sqrt{1-k^{2} s^{2}}} d s\right. \\
\left.\quad-(1-a) \int_{\eta_{a, k}}^{1 / k} \frac{s^{2}-m_{k}^{2}}{\sqrt{s^{2}-1} \sqrt{1-k^{2} s^{2}}} d s\right\} \\
=\eta_{a, k}^{-2}\left\{(1+a) \psi_{k}\left(\xi_{a, k}\right)-(1-a) \psi_{k}\left(\eta_{a, k}\right)\right\} \\
=t_{a, k} \eta_{a, k}^{-1}\left\{(1-a)+(1+a) \xi_{a, k} \eta_{a, k}^{-1}\right\} .
\end{aligned}
$$

Let $\left(k_{j}\right)_{j=1}^{\infty}$ be a sequence tending to 0 such that $\lim _{j \rightarrow \infty} k_{j} \eta_{a, k_{j}}(=d$, say) exists. Evidently, $0 \leq d \leq 1$. If $0<d<1$, then (29) shows that

$$
\lim _{j \rightarrow \infty} t_{a, k_{j}} \eta_{a, k_{j}}^{-1}=\frac{1}{\sqrt{1-d^{2}}}
$$

and hence

$$
\lim _{j \rightarrow \infty} k_{j} t_{a, k_{j}}=\frac{d}{\sqrt{1-d^{2}}}
$$

By (28), we have

$$
\lim _{j \rightarrow \infty} \xi_{a, k_{j}} k_{j} \log \left(1 / k_{j}\right)=\frac{\sqrt{1-d^{2}}}{d}
$$

By (30), we have 


$$
\frac{1}{d^{2}}\left\{(1+a)-(1-a) \sqrt{1-d^{2}}\right\}=\frac{1-a}{\sqrt{1-d^{2}}},
$$

which gives $d=2 \sqrt{a} /(1+a)$. We show that $d \neq 0,1$. Let $u(k)$ and $v(k)$ be the first quantity and the last quantity in (30), respectively. It holds that $u(k)=v(k) \quad(0<k<1)$. If $d=1$, then (29) shows that $\lim _{j \rightarrow \infty} v\left(k_{j}\right)=\infty$. We have

$$
\limsup _{j \rightarrow \infty} u\left(k_{j}\right) \leq \limsup _{j \rightarrow \infty}(1+a) \eta_{a, k_{j}}^{-2} m_{k_{j}}^{2} K\left(k_{j}^{\prime}\right)=(1+a),
$$

which contradicts (30). If $d=0$, then (29) shows that $\lim \sup _{j \rightarrow \infty} v\left(k_{j}\right)<$ $\infty$. By (28) and (29), we have

$$
\lim _{j \rightarrow \infty} \xi_{a, k_{j}} k_{j} \log \left(1 / k_{j}\right)=1 .
$$

Hence

$$
\begin{aligned}
\lim _{j \rightarrow \infty} u\left(k_{j}\right) & =\lim _{j \rightarrow \infty} \eta_{a, k_{j}}^{-2}\left\{(1+a) m_{k_{j}}^{2} \log \xi_{a, k_{j}}-(1-a) k_{j}^{-2}\right\} \\
& =2 a \lim _{j \rightarrow \infty} \eta_{a, k_{j}}^{-2} k_{j}^{-2}=\infty,
\end{aligned}
$$

which contradicts (30). Thus $d \neq 0,1$. Since $\left(k_{j}\right)_{j=1}^{\infty}$ is arbitrary as long as $\left(k_{j} \eta_{a, k_{j}}\right)_{j=1}^{\infty}$ converges, we obtain $\lim _{k \rightarrow 0} k \eta_{a, k}=d=2 \sqrt{a} /(1+a)$. Thus

$$
\lim _{k \rightarrow 0} k t_{a, k}=\frac{2 \sqrt{a} /(1+a)}{\sqrt{1-\left\{4 a /(1+a)^{2}\right\}}}=\frac{2 \sqrt{a}}{1-a} .
$$

Lemma 10. We have

$$
\lim _{k \rightarrow 0} t_{1, k} m_{k}^{-2}=\frac{1}{\sqrt{c^{2}-1}}
$$

where $c>0$ is the number satisfying

$$
c / \sqrt{c^{2}-1}=\log \left(c+\sqrt{c^{2}-1}\right) .
$$

Proof. Equalities (4) and (20) show that, with $\xi_{1, k}=\xi_{k}\left(t_{1, k}\right)$,

$$
\begin{aligned}
& \frac{1-\xi_{1, k}^{2} m_{k}^{-2}}{\sqrt{\xi_{1, k}^{2}-1} \sqrt{1-k^{2} \xi_{1, k}^{2}}}=t_{1, k} m_{k}^{-2}, \\
& \int_{1}^{\xi_{1, k}} \frac{1-s^{2} m_{k}^{-2}}{\sqrt{s^{2}-1} \sqrt{1-k^{2} s^{2}}} d s-t_{1, k} m_{k}^{-2} \xi_{1, k} \\
& \quad=m_{k}^{-2}\left\{\psi_{k}\left(\xi_{1, k}\right)-t_{1, k} \xi_{1, k}\right\}=m_{k}^{-2}\left\{\frac{\tau_{k}}{2}-\int_{0}^{t_{1, k}} \xi_{k}(s) d s\right\}=0,
\end{aligned}
$$

and hence 


$$
\frac{\left\{1-\xi_{1, k}^{2} m_{k}^{-2}\right\} \xi_{1, k}}{\sqrt{\xi_{1, k}^{2}-1} \sqrt{1-k^{2} \xi_{1, k}^{2}}}=\int_{1}^{\xi_{1, k}} \frac{1-s^{2} m_{k}^{-2}}{\sqrt{s^{2}-1} \sqrt{1-k^{2} s^{2}}} d s
$$

This shows that $\lim _{k \rightarrow 0} \xi_{1, k}=c$. Thus (31) yields the required equality.

LEMma 11. Let

$$
\Delta \gamma\left(z_{k}(t)\right)=\frac{\gamma\left(z_{k}(t)\right)-\left(1+x_{k}(t)\right) / 4}{y_{k}(t)} \quad(0<k<1, t \geq 0) .
$$

Then

$$
\begin{aligned}
\Delta \gamma\left(z_{k}(t)\right)= & \frac{k^{2} K\left(k^{\prime}\right)}{2 \pi} \int_{t}^{\infty}\left\{\eta_{k}(s)-\frac{s}{\sqrt{1+k^{2} s^{2}}}\right\} d s \\
& -\frac{k K\left(k^{\prime}\right)}{2 \pi} \sqrt{1+k^{2} t^{2}}
\end{aligned}
$$

Proof. We have

$$
\begin{aligned}
\Delta \gamma\left(z_{k}(t)\right)= & \frac{2 \gamma\left(z_{k}(t)\right)-1+\left(1-x_{k}(t)\right) / 2}{2 y_{k}(t)} \\
= & \frac{1}{2 y_{k}(t) l_{k}(t)}\left\{\frac{1-k}{k^{2}} \sqrt{1+k^{2} t^{2}}-l_{k}(t)+\frac{\tau_{k}}{2}-\int_{0}^{t} \xi_{k}(s) d s\right\} \\
= & \frac{k^{2} K\left(k^{\prime}\right)}{2 \pi}\left\{\frac{1-k}{k^{2}} \sqrt{1+k^{2} t^{2}}-\frac{\tau_{k}}{2}-\int_{0}^{t} \eta_{k}(s) d s\right\} \\
= & \frac{k^{2} K\left(k^{\prime}\right)}{2 \pi}\left\{\frac{1}{k^{2}} \sqrt{1+k^{2} t^{2}}-\frac{t}{k}-\frac{\tau_{k}}{2}+\int_{0}^{t}\left(\frac{1}{k}-\eta_{k}(s)\right) d s\right\} \\
& -\frac{k K\left(k^{\prime}\right)}{2 \pi} \sqrt{1+k^{2} t^{2}} \\
= & \frac{k^{2} K\left(k^{\prime}\right)}{2 \pi}\left\{\frac{1}{k^{2}} \sqrt{1+k^{2} t^{2}}-\frac{t}{k}-\int_{t}^{\infty}\left(\frac{1}{k}-\eta_{k}(s)\right) d s\right\} \\
& -\frac{k K\left(k^{\prime}\right)}{2 \pi} \sqrt{1+k^{2} t^{2}} \\
= & \frac{k^{2} K\left(k^{\prime}\right)}{2 \pi}\left\{\int_{t}^{\infty}\left(\frac{1}{k}-\frac{s}{\sqrt{1+k^{2} s^{2}}}\right) d s-\int_{t}^{\infty}\left(\frac{1}{k}-\eta_{k}(s)\right) d s\right\} \\
& -\frac{k K\left(k^{\prime}\right)}{2 \pi} \sqrt{1+k^{2} t^{2}} \\
= & \frac{k^{2} K\left(k^{\prime}\right)}{2 \pi} \int_{t}^{\infty}\left\{\eta_{k}(s)-\frac{s}{\sqrt{1+k^{2} s^{2}}}\right\} d s-\frac{k K\left(k^{\prime}\right)}{2 \pi} \sqrt{1+k^{2} t^{2}} .
\end{aligned}
$$

LEMMA $12 . \quad \gamma(z)=\frac{1}{2}+c_{k_{z}} \operatorname{Im} z \int_{\lambda(z)} \frac{\gamma(\zeta)^{2}}{\mathscr{L}(\zeta)\{\gamma(\zeta)+\mathscr{L}(\zeta)\}} h_{k_{z}}(\zeta) d(\operatorname{Im} \zeta)$ 
where $k_{z}$ is the first number in the pair associated with $z$ in (11),

$$
\begin{aligned}
c_{k} & =\frac{1}{4 \pi^{2} k}\left\{E\left(k^{\prime}\right)-k K\left(k^{\prime}\right)\right\}^{2}, \\
h_{k}(\zeta) & =\left\{\gamma(\zeta) \sqrt{\gamma(\zeta)^{2}+c_{k}^{\prime}(\operatorname{Im} \zeta)^{2}}+\gamma(\zeta)^{2}+c_{k}^{\prime \prime}(\operatorname{Im} \zeta)^{2}\right\}^{-1}, \\
c_{k}^{\prime} & =\frac{1}{4 \pi^{2}}(1-k)^{2} K\left(k^{\prime}\right)^{2}\left\{\left(k m_{k}^{2}-1\right)^{2}+2\left(k m_{k}^{2}-1\right)\right\}, \\
c_{k}^{\prime \prime} & =\frac{1}{4 \pi^{2}}(1-k)^{2} K\left(k^{\prime}\right)^{2}\left(k m_{k}^{2}-1\right) .
\end{aligned}
$$

Proof. Let $\zeta \in \lambda(z)$. Then $k_{\zeta}=k_{z}$ (=k, say). By (12), we have

$$
\begin{aligned}
& \frac{\gamma(\zeta)}{\mathscr{L}(\zeta)}-1=\frac{\gamma(\zeta)-\mathscr{L}(\zeta)}{\mathscr{L}(\zeta)} \\
= & \frac{2 \gamma(\zeta)^{2}}{\mathscr{L}(\zeta)\{\gamma(\zeta)+\mathscr{L}(\zeta)\}(1-k)^{2} t^{2}}\left\{\sqrt{\left(k t^{2}+m_{k}^{2}\right)^{2}+\left(k m_{k}^{2}-1\right)^{2} t^{2}}-\left(k t^{2}+m_{k}^{2}\right)\right\} \\
= & \frac{2 \gamma(\zeta)^{2}\left(k m_{k}^{2}-1\right)^{2}}{\mathscr{L}(\zeta)\{\gamma(\zeta)+\mathscr{L}(\zeta)\}(1-k)^{2}} \frac{1}{\sqrt{\left(k t^{2}+m_{k}^{2}\right)^{2}+\left(k m_{k}^{2}-1\right)^{2} t^{2}}+\left(k t^{2}+m_{k}^{2}\right)} .
\end{aligned}
$$

Since

$$
\begin{aligned}
& \sqrt{\left(k t^{2}+m_{k}^{2}\right)^{2}+\left(k m_{k}^{2}-1\right)^{2} t^{2}}+\left(k t^{2}+m_{k}^{2}\right) \\
= & \frac{1}{k}\left[\sqrt{\left\{\left(1+k^{2} t^{2}\right)+\left(k m_{k}^{2}-1\right)\right\}^{2}+\left(k m_{k}^{2}-1\right)^{2}\left(1+k^{2} t^{2}\right)-\left(k m_{k}^{2}-1\right)^{2}}\right. \\
& \left.+\left(1+k^{2} t^{2}\right)+\left(k m_{k}^{2}-1\right)\right] \\
= & \frac{1}{k}\left[\sqrt{1+k^{2} t^{2}} \sqrt{\left(1+k^{2} \iota^{2}\right)+\left(k m_{k}^{2}-1\right)^{2}+2\left(k m_{k}^{2}-1\right)}\right. \\
& \left.+\left(1+k^{2} t^{2}\right)+\left(k m_{k}^{2}-1\right)\right] \\
= & \frac{4 k^{4} l_{k}(t)^{2}}{k(1-k)^{2}}\left[\frac{(1-k) \sqrt{1+k^{2} t^{2}}}{2 k^{2} l_{k}(t)}\right. \\
& \times \sqrt{\frac{(1-k)^{2}\left(1+k^{2} t^{2}\right)}{4 k^{4} l_{k}(t)^{2}}+\frac{(1-k)^{2}\left\{\left(k m_{k}^{2}-1\right)^{2}+2\left(k m_{k}^{2}-1\right)\right\}}{4 k^{4} l_{k}(t)^{2}}} \\
& \left.+\frac{(1-k)^{2}\left(1+k^{2} t^{2}\right)}{4 k^{4} l_{k}(t)^{2}}+\frac{(1-k)^{2}\left(k m_{k}^{2}-1\right)}{4 k^{4} l_{k}(t)^{2}}\right] \\
= & \frac{4 \pi^{2}}{k(1-k)^{2} K\left(k^{\prime}\right)^{2}(\operatorname{Im} \zeta)^{2}}\left\{\gamma(\zeta) \sqrt{\gamma(\zeta)^{2}+c_{k}^{\prime}(\operatorname{Im} \zeta)^{2}}+\gamma(\zeta)^{2}+c_{k}^{\prime \prime}(\operatorname{Im} \zeta)^{2}\right\} \\
= & \frac{4 \pi^{2}}{k(1-k)^{2} K\left(k^{\prime}\right)^{2}(\operatorname{Im} \zeta)^{2}} h_{k}(\zeta)^{-1},
\end{aligned}
$$

we have 


$$
\begin{aligned}
\frac{1}{2} \int_{\lambda(z)} & \left\{\frac{\gamma(\zeta)}{\mathscr{L}(\zeta)}-1\right\} \frac{d(\operatorname{Im} \zeta)}{(\operatorname{Im} \zeta)^{2}} \\
= & \frac{k K\left(k^{\prime}\right)^{2}\left(k m_{k}^{2}-1\right)^{2}}{4 \pi^{2}} \int_{\lambda(z)} \frac{\gamma(\zeta)^{2}}{\mathscr{L}(\zeta)\{\gamma(\zeta)+\mathscr{L}(\zeta)\}} h_{k}(\zeta) d(\operatorname{Im} \zeta) \\
= & c_{k} \int_{\lambda(z)} \frac{\gamma(\zeta)^{2}}{\mathscr{L}(\zeta)\{\gamma(\zeta)+\mathscr{L}(\zeta)\}} h_{k}(\zeta) d(\operatorname{Im} \zeta),
\end{aligned}
$$

which gives the required equality.

We now give the proof of Theorem 8. Since

$$
\begin{aligned}
\Delta \gamma\left(z_{k}(0)\right) & =\frac{\gamma\left(z_{k}(0)\right)-1 / 4}{y_{k}(0)}=\frac{k^{2} K\left(k^{\prime}\right)}{\pi}\left\{\frac{1-k}{k^{2}}-\frac{\tau_{k}}{4}\right\} \\
& =\frac{K\left(k^{\prime}\right)}{\pi}\left\{1-k-\frac{k^{2} \tau_{k}}{4}\right\},
\end{aligned}
$$

we have (24). Let $0<a<1$. Then

$$
\begin{aligned}
& \frac{k^{2} K\left(k^{\prime}\right)}{2 \pi} \int_{t_{a, k}}^{\infty}\left\{\eta_{k}(s)-\frac{s}{\sqrt{1+k^{2} s^{2}}}\right\} d s \\
& \quad=\frac{K\left(k^{\prime}\right)}{2 \pi} \int_{k t_{a, k}}^{\infty}\left\{\eta_{k}^{*}(u)-\frac{u}{\sqrt{1+u^{2}}}\right\} d u,
\end{aligned}
$$

where

$$
\begin{aligned}
\eta_{k}^{*}(u)= & \frac{1}{\sqrt{2\left(1+u^{2}\right)}}\left[2 k^{2} m_{k}^{2}+\left(1+k^{2}\right) u^{2}\right. \\
& +\sqrt{\left.\left\{2 k^{2} m_{k}^{2}+\left(1+k^{2}\right) u^{2}\right\}^{2}-4\left(k^{4} m_{k}^{4}+k^{2} u^{2}\right)\left(1+u^{2}\right)\right]^{1 / 2}}
\end{aligned}
$$

Let $d_{k}=k^{2} m_{k}^{2}+k^{2}\left(m_{k}^{2}-1\right)\left(1-k^{2} m_{k}^{2}\right)\left(1-k^{2}\right)^{-1}$. Then we can write

$$
\begin{aligned}
\eta_{k}^{*}(u)= & \frac{1}{\sqrt{2\left(1+u^{2}\right)}}\left[2 k^{2} m_{k}^{2}+\left(1+k^{2}\right) u^{2}\right. \\
& +\sqrt{\left.\left(1-k^{2}\right)^{2} u^{4}+4\left\{k^{2} m_{k}^{2}\left(1+k^{2}\right)-\left(k^{2}+k^{4} m_{k}^{4}\right)\right\} u^{2}\right]^{1 / 2}} \\
= & \frac{u}{\sqrt{1+u^{2}}}\left[k^{2} m_{k}^{2} u^{-2}+\frac{1+k^{2}}{2}\right. \\
& \left.+\frac{1-k^{2}}{2} \sqrt{1+4 k^{2}\left(m_{k}^{2}-1\right)\left(1-k^{2} m_{k}^{2}\right)\left(1-k^{2}\right)^{-2} u^{-2}}\right]^{1 / 2} \\
= & \frac{u}{\sqrt{1+u^{2}}}\left[1+d_{k} u^{-2}\left\{1+d_{k} \omega_{1}(k, u)\right\}\right]^{1 / 2} \\
= & \frac{u}{\sqrt{1+u^{2}}}+\frac{d_{k}}{2 u \sqrt{1+u^{2}}}\left\{1+d_{k} \omega_{2}(k, u)\right\}
\end{aligned}
$$


with two functions $\omega_{j}(k, u)(j=1,2)$ satisfying $\sup \left|\omega_{j}(k, u)\right|<\infty$, where the supremum is taken over all pairs $(k, u)$ such that $0<k \leq 1 / 2$ and $u \geq \sqrt{a} /(1-a)$. Notice that $\lim _{k \rightarrow 0} d_{k}=0$ and $\lim _{k \rightarrow 0} d_{k} K\left(k^{\prime}\right)=2$. Thus Lemmas 9 and 11 show that

$$
\begin{aligned}
\gamma_{y}^{+}(a) & =\lim _{k \rightarrow 0} \Delta \gamma\left(z_{k}\left(t_{a, k}\right)\right) \\
& =\lim _{k \rightarrow 0}\left[\frac{k^{2} K\left(k^{\prime}\right)}{2 \pi} \int_{t_{a, k}}^{\infty}\left\{\eta_{k}(s)-\frac{s}{\sqrt{1+k^{2} s^{2}}}\right\} d s-\frac{k K\left(k^{\prime}\right)}{2 \pi} \sqrt{1+k^{2} t_{a, k}^{2}}\right] \\
& =\lim _{k \rightarrow 0} \frac{K\left(k^{\prime}\right)}{2 \pi} \int_{k t_{a, k}}^{\infty}\left\{\eta_{k}^{*}(u)-\frac{u}{\sqrt{1+u^{2}}}\right\} d u \\
& =\lim _{k \rightarrow 0} \frac{d_{k} K\left(k^{\prime}\right)}{4 \pi} \int_{k t_{a, k}}^{\infty} \frac{1}{u \sqrt{1+u^{2}}}\left\{1+d_{k} \omega_{2}(k, u)\right\} d u \\
& =\lim _{k \rightarrow 0} \frac{1}{2 \pi} \int_{2 \sqrt{a} /(1-a)}^{\infty} \frac{d u}{u \sqrt{1+u^{2}}}=\frac{1}{4 \pi} \log \frac{1}{a} .
\end{aligned}
$$

Thus (25) holds. Lemma 10 shows that $\lim _{k \rightarrow 0} k t_{1, k}=\infty$, and hence

$$
\begin{aligned}
& \lim _{k \rightarrow 0} \frac{k^{2} K\left(k^{\prime}\right)}{2 \pi} \int_{t_{1, k}}^{\infty}\left\{\eta_{k}(s)-\frac{s}{\sqrt{1+k^{2} s^{2}}}\right\} d s \\
& \quad=\lim _{k \rightarrow 0} \frac{d_{k} K\left(k^{\prime}\right)}{4 \pi} \int_{k t_{1, k}}^{\infty} \frac{1}{u \sqrt{1+u^{2}}}\left\{1+d_{k} \omega_{2}(k, u)\right\} d u=0 .
\end{aligned}
$$

By Lemmas 10 and 11, it follows that

$$
\begin{aligned}
\gamma_{y}^{+}(1) & =\lim _{k \rightarrow 0} \Delta \gamma\left(z_{k}\left(t_{1, k}\right)\right)=-\lim _{k \rightarrow 0} \frac{k K\left(k^{\prime}\right)}{2 \pi} \sqrt{1+k^{2} t_{1, k}^{2}} \\
& =-\frac{1}{2 \pi} \lim _{k \rightarrow 0} t_{1, k} m_{k}^{-2}=-\frac{1}{2 \pi \sqrt{c^{2}-1}}<0 .
\end{aligned}
$$

Thus (26) holds. Let $a>1$. Theorem 2 shows that

$$
\lim _{\zeta \rightarrow k_{a}, \zeta \in P} \frac{\gamma(\zeta)^{2}}{\mathscr{L}(\zeta)\{\gamma(\zeta)+\mathscr{L}(\zeta)\}} h_{k_{a}}(\zeta)=\frac{1}{4} .
$$

Thus Lemmas 7 and 12 yield that

$$
\gamma_{y}^{+}(a+i y)=\lim _{y \downarrow 0} \frac{\gamma(a+i y)-1 / 2}{y}=-\frac{1}{4} c_{k_{a}} \lim _{y \downarrow 0} \int_{0}^{y} d s=0
$$

and

$$
\gamma_{y y}^{+}(a)=2 \lim _{y \downarrow 0} \frac{\gamma(a+i y)-1 / 2}{y^{2}}=-\frac{1}{2} c_{k_{a}} \lim _{y \downarrow 0} \frac{1}{y} \int_{0}^{y} d s
$$




$$
\begin{aligned}
& =-\frac{1}{2} c_{k_{a}}=-\frac{1}{8 \pi^{2} k_{a}}\left\{E\left(k_{a}^{\prime}\right)-k_{a} K\left(k_{a}^{\prime}\right)\right\}^{2} \\
& =-\frac{1}{8 \pi^{2}} \frac{a+1}{a-1}\left\{E\left(\frac{2 \sqrt{a}}{a+1}\right)-\frac{a-1}{a+1} K\left(\frac{2 \sqrt{a}}{a+1}\right)\right\}^{2},
\end{aligned}
$$

which shows (27). This completes the proof of Theorem 8 .

\section{$\S 5$. The constant $\sigma_{0}$}

In this section, we study the following extremum problem: $\sigma_{0}=$ $\inf \gamma(x+i y) / \gamma(x)$, where the infimum is taken over all real numbers $x$ and $y$. We show

TheOREm 13. Let $\rho(a)=\min _{y \geq 0} \gamma(a+i y) / \gamma(a)(a \geq 0)$. Then $\sigma_{0}=\rho(1)$ and $\sigma_{0}<\rho(a)(a \neq 1)$.

Here is a lemma necessary for the proof.

Lemma 14. For each $0<k<1$,

(32) $\quad \gamma\left(z_{k}(t)\right)$ is strictly increasing,

(33) $4 \gamma\left(z_{k}(t)\right) /\left(1+x_{k}(t)\right)$ is strictly decreasing.

Proof. Theorem 1 shows that

$$
\gamma\left(z_{k}(t)\right)=\frac{1}{2}+\frac{y_{k}(t)}{2} \int_{t}^{\infty}\left\{\frac{\gamma\left(z_{k}(s)\right)}{\mathscr{L}\left(z_{k}(s)\right)}-1\right\} \frac{y_{k}^{\prime}(s)}{y_{k}(s)^{2}} d s
$$

and hence

$$
\begin{aligned}
\frac{d}{d t} \gamma\left(z_{k}(t)\right)= & \frac{y_{k}^{\prime}(t)}{2} \int_{t}^{\infty}\left\{\frac{\gamma\left(z_{k}(s)\right)}{\mathscr{L}\left(z_{k}(s)\right)}-1\right\} \frac{y_{k}^{\prime}(s)}{y_{k}(s)^{2}} d s \\
& -\frac{y_{k}(t)}{2}\left\{\frac{\gamma\left(z_{k}(t)\right)}{\mathscr{L}\left(z_{k}(t)\right)}-1\right\} \frac{y_{k}^{\prime}(t)}{y_{k}(t)^{2}} .
\end{aligned}
$$

Thus Theorem 2 and (17) yield (32). Since

$$
\begin{aligned}
\frac{1+x_{k}(t)}{4} & =\frac{1}{2 l_{k}(t)}\left\{l_{k}(t)-\frac{\tau_{k}}{2}+\int_{0}^{t} \xi_{k}(s) d s\right\} \\
& =\frac{1}{2 l_{k}(t)}\left\{\frac{\tau_{k}}{2}+\int_{0}^{t} \eta_{k}(s) d s\right\}=\frac{1}{2 l_{k}(t)}\left\{\psi_{k}\left(\eta_{k}(t)\right)+t \eta_{k}(t)\right\}
\end{aligned}
$$

we have, by (6), 
(34)

$$
\begin{aligned}
\frac{d}{d t} \frac{4 \gamma\left(z_{k}(t)\right)}{1+x_{k}(t)}= & \left.\frac{1-k}{k^{2}} \frac{d}{d t} \frac{\sqrt{1+k^{2} t^{2}}}{\psi_{\cdot k}\left(\eta_{k}(t)\right)+t \eta_{k}(t)}\right] \\
= & \frac{1-k}{k^{2}\left\{\psi_{k}\left(\eta_{k}(t)\right)+t \eta_{k}(t)\right\}^{2}} \\
& \times\left[\frac{k^{2} t}{\sqrt{1+k^{2} t^{2}}}\left\{\psi_{\psi_{k}}\left(\eta_{k}(t)\right)+t \eta_{k}(t)\right\}-\sqrt{1+k^{2} t^{2}} \eta_{k}(t)\right] \\
= & \frac{1-k}{k^{2} \sqrt{1+k^{2} t^{2}}\left\{\psi_{k}\left(\eta_{k}(t)\right)+t \eta_{k}(t)\right\}^{2}}\left\{k^{2} t \psi_{k}\left(\eta_{k}(t)\right)-\eta_{k}(t)\right\}
\end{aligned}
$$

Since $m_{k}>1$, we have, with $\eta=\eta_{k}(t)$,

$$
\begin{aligned}
k^{2} t_{\psi_{k}}(\eta) & =k^{2} t\left\{\psi_{\cdot k}(\eta)-\psi_{k}(1 / k)\right\} \\
& =\frac{k^{2}\left(\eta^{2}-m_{k}^{2}\right)}{\sqrt{\eta^{2}-1} \sqrt{1-k^{2} \eta^{2}}} \int_{\eta}^{1 / k} \frac{s^{2}-m_{k}^{2}}{\sqrt{s^{2}-1} \sqrt{1-k^{2} s^{2}}} d s \\
& <\frac{k^{2} \eta}{\sqrt{1-k^{2} \eta^{2}}} \int_{\eta}^{1 / k} \frac{s}{\sqrt{1-k^{2} s^{2}}} d s=\eta .
\end{aligned}
$$

Hence the first quantity in (34) is negative, which gives (33).

We now give the proof of Theorem 13. Let $a>1$. Since $\lim _{y \rightarrow \infty} \gamma(a+i y) / \gamma(a)=1$, there exists $y_{a} \geq 0$ such that

$$
\rho(a)=\gamma\left(a+i y_{a}\right) / \gamma(a)=2 \gamma\left(a+i y_{a}\right) .
$$

By (27), we have $y_{a}>0$. Hence there exists a pair $\left(k^{0}, t^{0}\right)$ such that $a+i y_{a}=z_{k^{0}}\left(t^{0}\right)$. Let $t^{1}>0$ be the number such that $x_{k 0}\left(t^{1}\right)=1$. Then $t^{1}<t^{0}$. Hence, by (32), it follows that

$$
\rho(1) \leq \gamma\left(z_{k 0}\left(t^{1}\right)\right) / \gamma(1)=2 \gamma\left(z_{k 0}\left(t^{1}\right)\right)<2 \gamma\left(z_{k 0}\left(t^{0}\right)\right)=\rho(a) .
$$

Inequality (26) shows that $\rho(1)<1$. Let $0 \leq a<1$. Then there exists $y_{a} \geq 0$ such that

$$
\rho(a)=\frac{\gamma\left(a+i y_{a}\right)}{\gamma(a)}=\frac{4 \gamma\left(a+i y_{a}\right)}{1+a} .
$$

If $y_{a}=0$, then $\rho(1)<1=\rho(a)$. If $y_{a}>0$, then there exists a pair $\left(k^{0}, t^{0}\right)$ such that $a+i y_{a}=z_{k 0}\left(t^{0}\right)$. Let $t^{1}>0$ be the number such that $x_{k 0}\left(t^{1}\right)=1$. Then $t^{1}>t^{0}$. Hence, by (33), it follows that

$$
\begin{aligned}
\rho(1) & \leq 4 \gamma\left(z_{k^{0}}\left(t^{1}\right)\right) /\left(1+x_{k 0}\left(t^{1}\right)\right) \\
& <4 \gamma\left(z_{k^{0}}\left(t^{0}\right)\right) /\left(1+x_{k 0}\left(t^{0}\right)\right)=\rho(a) .
\end{aligned}
$$

Thus 


$$
\rho(1)=\min _{a \geq 0} \rho(a), \quad \rho(1)<\rho(a) \quad(a \neq 1) .
$$

which gives the required inequalities in Theorem 13. This completes the proof of Theorem 13.

From the point of view of Vitushkin-Garnett's example, it is interesting to estimate $\sigma_{0}$. A rough estimate is given as follows. The Garabedian function [2, p. 19] of an interval [-1/2,1/2] is given by

$$
\psi(\zeta)=\frac{1}{2}\left\{1+\frac{\zeta}{\sqrt{\zeta^{2}-(1 / 4)}}\right\}
$$

in fact,

$$
\frac{1}{2 \pi} \int_{\partial[-1 / 2,1 / 2]}|\psi(\zeta)||d \zeta|=\frac{1}{4 \pi} \int_{-1 / 2}^{1 / 2} \frac{d s}{\sqrt{(1 / 4)-s^{2}}}=\frac{1}{4} .
$$

Since $\psi(\zeta) \psi(\zeta+1+i y)$ is analytic outside $\Gamma(1+i y)$ and equal to 1 at infinity, we have

$$
\gamma(1+i y) \leq \frac{1}{2 \pi} \int_{\partial \Gamma(1+i y)}|\psi(\zeta) \psi(\zeta+1+i y) \| d \zeta| \quad \text { (cf. [2, p. 19]) }
$$

Thus Theorem 13 shows that

$$
\sigma_{0} \leq \inf _{y \geq 0} \frac{1}{\pi} \int_{\partial \Gamma(1+i y)}|\psi(\zeta) \psi(\zeta+1+i y)||d \zeta| .
$$

We can easily compute the right-hand side of (35). The estimate by this method is rough, however, this method gives a new approach to the construction of sets of Vitushkin-Garnett type (cf. [8, p. 81]). In order to get a better estimate, it is necessary to study, in detail, incomplete elliptic integrals. Recall that

$$
\begin{aligned}
\sigma_{0} & =\min _{0<k<1} 2 \gamma\left(z_{k}\left(t_{1, k}\right)\right), \\
\gamma\left(z_{k}(t)\right) & =\left\{\frac{1-k}{2 k} \sqrt{t^{2}+k^{-2}}\right\} / l_{k}(t), \\
l_{k}(t) & =\psi_{k}\left(\eta_{k}(t)\right)+\psi_{k}\left(\xi_{k}(t)\right)+t\left\{\eta_{k}(t)-\xi_{k}(t)\right\}, \\
\psi_{\cdot k}(x) & =\int_{1}^{x} \frac{m_{k}^{2}-s^{2}}{\sqrt{s^{2}-1} \sqrt{1-k^{2} s^{2}}} d s \quad(1 \leq x \leq 1 / k) .
\end{aligned}
$$

Since

$$
\psi_{k}(x)=-\psi_{\cdot k}(1 / k)+\psi_{k}(x)=\int_{x}^{1 / k} \frac{s^{2}-m_{k}^{2}}{\sqrt{s^{2}-1} \sqrt{1-k^{2} s^{2}}} d s
$$


we have, by making the substitution $1-k^{2} s^{2}=k^{2} u^{2}$,

$$
\begin{aligned}
\psi_{k}(x) & =k^{-2} \int_{0}^{\nu(x)} \sqrt{\frac{1-k^{\prime 2} u^{2}}{1-u^{2}}} d u-m_{k}^{2} \int_{0}^{\nu(x)} \frac{d u}{\sqrt{1-u^{2}} \sqrt{1-k^{\prime 2} u^{2}}} \\
& =k^{-2} E\left(\arcsin \nu(x), k^{\prime}\right)-m_{k}^{2} F\left(\arcsin \nu(x), k^{\prime}\right),
\end{aligned}
$$

where $\nu(x)=\sqrt{1-k^{2} x^{2}} / k^{\prime}$. Thus $\psi_{k}(x)$ can be computed with the aid of Landen's transformation [4, p. 250] or Jacobian theta functions [4, p. 292]. (As is well known, Landen's transformation yields that

$$
\begin{aligned}
& F\left(\varphi, k^{\prime}\right)=\frac{1}{1+k} F\left(\psi, \frac{1-k}{1+k}\right), \\
& E\left(\varphi, k^{\prime}\right)=-\frac{k(1+k)}{2} F\left(\varphi, k^{\prime}\right)+\frac{1+k}{2} E\left(\psi, \frac{1-k}{1+k}\right)+\frac{1-k}{2} \sin \psi,
\end{aligned}
$$

where $\psi$ is defined by $\tan (\psi-\varphi)=k \tan \varphi$. Since $(1-k) /(1+k)<k^{\prime}$, we can compute $E\left(\varphi, k^{\prime}\right)$ and $F\left(\varphi, k^{\prime}\right)$ by repeating this formula.) Equality (20) for $a=1$ can be rewritten as

$$
0=\frac{\tau_{k}}{2}-\int_{0}^{t_{1, k}} \xi_{k}(s) d s=\psi_{k}\left(\xi_{k}\left(t_{1, k}\right)\right)-t_{1, k} \xi_{k}\left(t_{1, k}\right)
$$

and hence

$$
m_{k} t_{1, k}=t_{1, k}\left\{m_{k}-\xi_{k}\left(t_{1, k}\right)\right\}+\psi_{k}\left(\xi_{k}\left(t_{1, k}\right)\right) .
$$

We now inductively define a sequence $\left(t_{1, k}^{(n)}\right)_{n=0}^{\infty}$ by $t_{1, k}^{(0)}=0$,

$$
m_{k} t_{1, k}^{(n)}=t_{1, k}^{(n-1)}\left\{m_{k}-\xi_{k}\left(t_{1, k}^{(n-1)}\right)\right\}+\psi_{k k}\left(\xi_{k}\left(t_{1, k}^{(n-1)}\right)\right) \quad(n \geq 1) .
$$

Since

$$
t\left\{m_{k}-\xi_{k}(t)\right\}+\psi_{k}\left(\xi_{k}(t)\right)=\frac{\tau_{k}}{2}+\int_{0}^{t}\left\{m_{k}-\xi_{k}(s)\right\} d s
$$

we have

$$
\begin{aligned}
m_{k}\left|t_{1, k}^{(n)}-t_{1, k}^{(n-1)}\right| & =\left|\int_{t_{1, k}^{(n-2)}}^{t_{1, k}^{(n-1)}}\left\{m_{k}-\xi_{k}(s)\right\} d s\right| \\
& \leq\left(m_{k}-1\right)\left|t_{1, k}^{(n-1)}-t_{1, k}^{(n-2)}\right| \quad(n \geq 2),
\end{aligned}
$$

and hence

$$
\left|t_{1, k}-t_{1, k}^{(n)}\right| \leq \sum_{l=n}^{\infty}\left(1-m_{k}^{-1}\right)^{l}\left|t_{1, k}^{(1)}\right|=\frac{m_{k} \tau_{k}}{2}\left(1-m_{k}^{-1}\right)^{n} \quad(n \geq 0) .
$$


This shows that $\left(t_{1, k}^{(n)}\right)_{n=0}^{\infty}$ converges to $t_{1, k}$. (In the case where $k$ is small, the speed of the convergence of $\left(t_{1, k}^{(n)}\right)_{n=0}^{\infty}$ is slow. Hence, by using $\left(t_{1, k}^{(n)}\right)_{n=0}^{\infty}$, we choose first $\tilde{t}_{1, k}$ sufficiently near to $t_{1, k}$ and define next $\left(\tilde{t}_{1, k}^{(n)}\right)_{n=0}^{\infty}$ by $\tilde{t}_{1, k}^{(0)}=\tilde{t}_{1, k}$,

$$
\tilde{t}_{1, k}^{(n)}=\tilde{t}_{1, k}^{(n-1)}\left\{1-\varepsilon_{k} \xi_{k}\left(\tilde{t}_{1, k}^{(n-1)}\right)\right\}+\varepsilon_{k} \psi_{k}\left(\xi_{k}\left(\tilde{t}_{1, k}^{(n-1)}\right)\right) \quad(n \geq 1),
$$

where $\varepsilon_{k}>0$ is chosen so that the convergence of $\left(\tilde{t}_{1, k}^{(n)}\right)_{n=0}^{\infty}$ is rapid. Notice that $t_{1, k}=\lim _{n \rightarrow \infty} \tilde{t}_{1, k}^{(n)}$. $) \quad$ Thus we can compute $2 \gamma\left(z_{k}\left(t_{1, k}\right)\right)(0<k<1)$. The author expresses his thanks to Prof. Yonezawa and Mr. Sakurai who practiced our program. Prof. Yonezawa shows that $0.95 \leq \sigma_{0} \leq 0.97$. ( $\sigma_{0}$ is attained when $k$ is near to 0.1.)

\section{REFERENCES}

[1] C. Ferrari, Sulla transformazione conforme di due cerchi in due profili alari, Memorie della R. Accad. delle Scienze di Torino, Serie II, 67 (1930), 1-15.

[ 2 ] J. Garnett, Analytic capacity and measure, Lecture Notes in Math., 297, SpringerVerlag, Berlin, 1972.

[ 3 ] E. Garrick, Potential flow about arbitrary biplane wing sections, Technical Report No. 542, N.A.C.A. (1936), 47-75.

[ 4 ] H. Hancock, Lectures on the theory of elliptic functions, Dover, New York, 1958.

[ 5 ] Y. Komatu, (Japanese), Theory of conformal mappings II, Kyoritsu, Tokyo, 1947.

[ 6 ] B. B. Mandelbrot, The fractal geometry and nature, Freeman, San Francisco, 1982.

[ 7 ] L. M. Milne-Thomson, Theoretical hydrodynamics, Fifth edition, Macmillan, London, 1968.

[ 8 ] T. Murai, A real variable method for the Cauchy transform, and analytic capacity, Lecture Notes in Math., 1307, Springer-Verlag, Berlin, 1988.

[9] - The power $3 / 2$ appearing in the estimate of analytic capacity, Pacific J. Math., 143 (1990), 313-340.

[10] Z. Nehari, Conformal mapping, McGraw-Hill, New York, 1952.

[11] Ch. Pommerenke, Über die analytische Kapazität, Ark. der Math., 11 (1960), 270277.

[12] L. Sario and K. Oikawa, Capacity functions, Springer-Verlag, Berlin, 1969.

[13] T. Sasaki, (Japanese), Applications of conformal mappings, Fuzanbo, Tokyo, 1939.

[14] Vitushkin, (Russian), Example of a set of positive length but of zero analytic capacity, Dokl. Akad. Nauk. SSSR, 127 (1959), 246-249.

Department of Mathematics

School of Science

Nagoya University

Nagoya, 464-01

Japan 